\title{
Spinecor vs sport brace for adolescent idiopathic scoliosis: short term results
}

\author{
Fabio Zaina*, Claudia Fusco, Michele Romano, Alessandra Negrini, Stefano Negrini \\ From 7th International Conference on Conservative Management of Spinal Deformities \\ Montreal, Canada. 20-22 May 2010
}

\begin{abstract}
Introduction
SpineCor and rigid braces have both results testifying their effectiveness in Adolescent Idiopathic Scoliosis (AIS) treatment: an RCT recently compared the two showing the superiority of rigid braces. Since some years we introduced SpineCor as a treatment for patients at curves between 20 and 30 Cobb degrees to improve the quality of life and reduce rigid bracing. The objective of this study is to compare the short term results of the Spinecor vs SPoRT brace for AIS in this selected population.
\end{abstract}

\section{Materials and methods}

Study design: retrospective controlled study

\section{Population}

103 consecutive AIS patients from our prospective database ( 85 female; age $13 \pm 1$, Cobb $24 \pm 5^{\circ}$; ATR $8 \pm 4^{\circ}$ ): 29 patients (19 females; age 13 \pm 1 ; TRACE score 6; Cobb angle $22 \pm 5^{\circ}$; ATR $9 \pm 3^{\circ}$, Risser $0-3$ ) treated by Spinecor $20 / 24$ hours per day; 74 patients (66 females; age $13 \pm 1$; TRACE score 5; Cobb angle $25 \pm 4^{\circ}$; ATR $8 \pm 4^{\circ}$, Risser $0-3)$ treated by SPoRT Brace 18 to 23 hours per day.

The short term results of treatment were evaluated at the moment of the first X-ray performed without the Spinecor $17 \pm 4$ months later. For the SPoRT Brace group the $\mathrm{X}$-ray were performed after $18 \pm 4$. Patients were evaluated both clinically and radiographically before and after the treatment. Main outcome measures: TRACE, Cobb angle (changes $> \pm 4$ ), ATR (changes $> \pm 2$ ).

\section{Results}

The two groups had no statistically significant differences at baseline apart from the Cobb angles, worst in the SPoRT Group. The Median value of TRACE didn't change for SPoRT while decreased for Spinecor (5 vs 4) but the change was not significant (NS). Considering the number of patients aesthetical changes were similar for the SPoRT (34\% improved, 58\% stable and $8 \%$ worsened) and the SpineCor (55\%, $41 \%$ and $4 \%$ ).

Considering the number of patients changed $> \pm 5^{\circ}$ Cobb in the SPoRT Group there was $42 \%$ improved, $40 \%$ stable and $18 \%$ worsened vs $28 \%, 52 \%$ and $21 \%$ respectively in SpineCor (NS). Considering Mean Cobb angle, there was animprovement of $3^{\circ}$ vs $0^{\circ}(\mathrm{p}<0.05)$ for SPoRT. For ATR there was a significant improvement only in the SPoRT Group ( $\mathrm{p}<0.05)$.

\section{Discussion}

Both the treatment showed to be effective in improving the Aesthetics in AIS of 20-30 Cobb. For the other parameters, the SPoRT brace seems to be slightly more effective than the SpineCor both for the Cobb angle and the ATR. Nevertheless, both treatments were effective in preventing the evolution of the curves. The main limits of the study are the retrospective design, the small population and the short term evaluation.

\section{Conclusion}

Short term results showed a similar efficacy for Aesthetics of both treatments with better results of the SPoRT Brace for Cobb Angle and ATR in 20 to $30^{\circ}$ Cobb curves.

Published: 10 September 2010

doi:10.1186/1748-7161-5-S1-O55

Cite this article as: Zaina et al.: Spinecor vs sport brace for adolescent idiopathic scoliosis: short term results. Scoliosis 2010 5(Suppl 1):055. 